\title{
Bundeseinheitliche Rufnummer für den ärztlichen Bereitschaftsdienst kommt
}

— Die ärztlichen Bereitschaftsdienste der einzelnen KVen können zur Zeit in Deutschland nur über mehrere hundert verschiedene Telefonnummern erreicht werden. Die Vertreterversammlung der KBV hatte deshalb bereits 2007 beschlossen, die bundesweit einheitliche Rufnummer 116117 einzuführen. Nachdem die Europäische Kommission entschieden hatte, dass in den EU-Mitgliedstaaten die mit 116 beginnenden nationalen Nummern für Telefondienste mit sozialem Wert genutzt werden dürfen, war der Weg frei.

\section{MMW Kommentar}

Die einheitliche Nummer bringt insbesondere den Bürgern Vorteile. Ein Anruf wird automatisch an den zuständigen Bereitschaftsdienstbereich weitergeleitet - je nach regionaler Organisation des Bereitschaftsdienstes der jeweiligen Kassenärztlichen Vereinigung zu einem dienst- habenden Arzt, einem KV-Callcenter, einer $K V$-Bezirksstelle oder an die weiteren externen Kooperationspartner. Patientenanrufe, die nicht eindeutig einem Bereitschaftsdienstbezirk zuzuordnen sind, werden automatisch an ein bundesweit zuständiges Callcenter geleitet. Geplant ist, dass die bundesweite Nummer 2011 an den Start geht. Derzeit bereitet die KBV eine europaweite Ausschreibung zur technischen Umsetzung vor.

\section{KBV legt Vorschläge zur neuen Bedarfsplanung vor}

— Die KBV hat Vorschläge zur Gesetzesänderung bei der Bedarfsplanung vorgelegt. Ziel der Maßnahme soll eine bessere Verteilung der noch vorhandenen Ärztinnen und Ärzte in den einzelnen Regionen Deutschlands sein. So sollen bei den KVen Strukturfonds zur finanziellen Unterstützung von Niederlassungen auf ausgeschriebenen Versorgungssitzen in Regionen mit notwendigem Versorgungsbedarf verwaltet werden.

Gedacht ist dabei vor allem an Zuschüsse zur Finanzierung von Praxen und der Organisation des Notfall- und Bereitschaftsdienstes sowie von sog. Arztstationen in strukturschwachen Gebieten. Soweit trotz derartiger Angebote keine Bewerbungen erfolgen, schlägt die KBV vor, dass die betroffenen KVen Eigeneinrichtungen etwa durch MVZ oder ärztliche Versorgungszentren, in denen von der KV angestellte Ärzte bzw. Psychotherapeuten arbeiten, schaffen. Auch ist daran gedacht, dass vorhandene ärztliche bzw. psychotherapeutische Praxen und MVZ unter Mitwirkung von Land und Kommune die neu zu schaffenden Arztstationen in der Weise betreuen, dass gegebenenfalls stundenweise die hausärztliche und fachärztliche Grundversorgung gewährleistet wird („Filialisierung“).

Durch eine immer präsente und qualifizierte Fachkraft soll aus einer solchen Arztstation aber auch die ,aufsuchende Betreuung" mitorganisiert werden (Modellprojekte Schwester Agnes, Verah). Medizinische Versorgungszentren (MVZ) sollen von den KVen allein oder gemeinsam mit Krankenhäusern gegründet werden. Vertragsärzte, Vertragspsychotherapeuten und Krankenhausärzte übernehmen darin als Brückenfunktionen u.a. die nachstationäre ambulante Versorgung, die Errichtung von Notfallambulanzen in unterversorgten Gebieten sowie die bis-

\section{$\mathrm{Abb}$}

KBV, DKG und GKV-SV regeln die Zusammenarbeit zwischen

\begin{tabular}{|c|c|c|}
\hline $\begin{array}{c}\text { Ver- } \\
\text { trags- } \\
\text { ärzten }\end{array}$ & $\begin{array}{c}\text { Vertrags- } \\
\text { psycho- } \\
\text { thera- } \\
\text { peuten }\end{array}$ \\
\end{tabular}

Im Verbund mit Krankenhausgesellschaft und Kassen will die KBV die Ärzteverteilung in der Fläche verbessern. herigen Aufgaben der Praxiskliniken. Der Betrieb solcher Einrichtungen kann nach Vorstellung der KBV durch die Vermittlung freiberuflich tätiger Vertragsärzte (Konsiliarärzte) und Psychotherapeuten sowie durch Honorarärzte (keine Vertragsärzte) erfolgen.

\section{MMW Kommenta}

Die Pläne der KBV sind zweifelsohne revolutionär, erklären zugleich aber auch die Initiative im Zusammenhang mit der Beteiligung an der Patiomed AG. Bedenklich ist die geplante Umlenkung der Ärzte von überversorgten in unterversorgte Gebiete. So ist geplant, in überversorgten Gebieten Arztsitze oder Psychotherapeutensitze nicht mehr neu zu besetzen, wenn sich der betreffende Vertragsarzt bzw. Psychotherapeut zur Ruhe setzen will. In diesem Fall ist vorgesehen, dass die KV die Praxis zum Verkehrswert aufkauft und der aufgekaufte Arztsitz nicht mehr besetzt wird. Dies ist sicherlich ein zentraler Punkt, dessen Umsetzung von fundamentaler Bedeutung für das Gelingen eines solchen Projektes ist. Fraglich dürfte sein, ob eine solche Vorgehensweise einer verfassungsrechtlichen Überprüfung stand hält. 